\title{
Consommation durable et sécurité alimentaire
}

\author{
Coralie BONNIN-DE TOFFOLI
}

Enseignante à Skema Business School

Chercheur associé au CREDECO (GREDEG CNRS UMR 7321)

et Nathalie LAZARIC

Université de Nice Sophia Antipolis, CNRS GREDEG (GREDEG CNRS UMR 7321)

Les prémices de la consommation durable sont apparus dès les années soixante-dix. En effet, les graves catastrophes environnementales ${ }^{1}$, la crise du pétrole et le rejet de la société de consommation ont contribué à la reconnaissance du lien étroit entre l'humain et l'environnement. La genèse d'une réflexion sur la consommation durable émerge alors avec la formulation du concept de développement durable par le Rapport BRUNDTLAND ${ }^{2}$. Cependant, il faudra attendre l'adoption de la Déclaration de Rio en 1992 pour consacrer la consommation durable comme une priorité environnementale ${ }^{3}$ et de développement social ${ }^{4}$. Dans la décennie qui a suivi, diverses actions ont été menées pour assurer une consommation durable, toutefois leur succès reste relativement mitigé. Tout d'abord, l'absence d'une définition uniforme et claire du concept de «consommation durable » a freiné l'adoption de mesures efficaces et cohérentes à l'échelle nationale ${ }^{5}$. Par ailleurs, la fragmentation des diverses approches nationales, l'absence de cohérence des moyens financiers et des mesures

\footnotetext{
${ }^{1}$ En 1978, les côtes bretonnes ont été touchées par le naufrage du pétrolier Amoco-Cadiz; en 1979, l'entreprise pharmaceutique Seveso en Italie a émis des émanations toxiques de dioxine ; en 1984, l'Inde est victime d'une grave explosion dans une usine de pesticides à Bhopal ; en 1986, un accident nucléaire survient dans une centrale nucléaire à Tchernobyl.

${ }^{2}$ COMMISSION MONDIALE SUR L'ENVIRONNEMENT ET LE DEVELOPPEMENT, Notre avenir à tous, (ci-après cité « Rapport BRUNDTLAND »), St-Jean-sur-Richelieu (Qc), Lambda, 1987, 458 p.

${ }^{3}$ J. MURPHY et M. J. COHEN, «Consumption, Environment and Public Policy », in J. MURPHY et M. J. COHEN (dir.), Exploring Sustainable Consumption: Environmental Policy and the Social Sciences, 2001, Oxford, Pergamon, p. 3 ; N. ROBINS et S. ROBERTS, « Consumption in a Sustainable World », Workshop, 2-4 juin 1998, Kabelvaag, Norvège.

${ }_{4}^{4}$ R. ROMI, G. BOSSIS et S. ROUSSEAUX, Droit international et européen de l'environnement, Paris, Montchrestien, 2005, p. 14.

5 Plusieurs approches définissent la consommation durable. UNCSD, International Work Programme, 1995 ; Symposium on Sustainable Consumption, Oslo, Norvège, 19-20 janvier 1994 ; E. G. FALKMAN, Waste Management International Sustainable Production and Consumption : A Business Perspective, World Business Council for Sustainable Development (WBCSD) ; N. ROBINS et S. ROBERTS, Changing Consumption and Production Patterns : Unlocking Trade Opportunities, International Institute for Environment and Development et United Nations Department of Policy Coordination and Sustainable Development, 1997 ; E. SALIM, The challenge of sustainable consumption as seen from the South, Symposium: Sustainable Consumption, Oslo, Norvège, 19-20 janvier 1994. Nous retiendrons la définition adoptée par les Principes directeurs sur la protection du consommateur qui prévoit que «La consommation durable devrait notamment permettre de satisfaire les besoins en biens et services des générations actuelles et à venir, suivant des modalités qui puissent s'inscrire dans le long terme du point de vue économique, social et écologique » (par. 42).
} 
adoptées représentent des limites sérieuses à l'adoption de modes de consommation durables ${ }^{6}$. La Commission européenne a adopté un Plan d'action pour une consommation et une production durables et pour une politique industrielle durable en $2008^{7}$ afin de promouvoir une politique européenne en faveur de la consommation durable applicable à l'ensemble des Etats membres. Toutefois, cette politique souffre de certaines lacunes car les mesures adoptées ne permettent pas toujours d'atteindre les objectifs annoncés ${ }^{8}$.

La sécurité alimentaire et la consommation durable sont étroitement liées au concept de développement durable. Ainsi, le Rapport BRUNDTLAND reconnaît la sécurité alimentaire et l'adoption de modes de consommation plus respectueux de l'environnement comme des priorités pour assurer le développement durable ${ }^{9}$. Notons que la sécurité alimentaire « existe lorsque tous les êtres humains ont à tout moment, un accès physique et économique à une nourriture suffisante, saine et nutritive leur permettant de satisfaire leurs besoins énergétiques et leurs préférences alimentaires pour mener une vie saine et active ${ }^{10}$. La sécurité alimentaire couvre désormais les aspects non seulement quantitatifs, mais aussi qualitatifs de la consommation d'aliments. Il est donc essentiel de promouvoir une production et une consommation durables pour permettre aux consommateurs et aux producteurs de faire des choix durables afin de satisfaire les besoins essentiels des populations ${ }^{11}$. Avec le concept du développement durable, il est devenu important d'intégrer les impératifs environnementaux et les besoins sociaux lors de la prise de décision en matière de développement économique afin d'assurer la pérennité des écosystèmes. Il y a donc une interdépendance entre les facteurs économique, écologique et social. Il est dès lors essentiel d'adopter des modes de production et de consommation viables pour pouvoir nourrir en quantité et qualité suffisante la population mondiale.

\footnotetext{
${ }^{6}$ Rapport BRUNDTLAND.

${ }^{7} \mathrm{CE}$, Communication de la Commission, Plan d'action pour une consommation et une production durables et pour une politique industrielle durable, p. 4, [COM(2008) 397 final].

${ }^{8}$ Le label écologique n'est indiqué que sur un nombre limité de produits (sont exclus les produits alimentaires, les boissons et les médicaments). Par ailleurs, les informations relatives à l'efficacité énergétique qui doivent être communiquées aux consommateurs ne concernent que les appareils ménagers et les équipements de bureau (Directive 92/75/CEE sur l'étiquetage de la consommation en énergie et le Règlement 106/2008 concernant un programme communautaire d'étiquetage relatif à l'efficacité énergétique des équipements de bureau).

${ }^{9}$ Rapport BRUNDTLAND.

${ }^{10}$ Plan d'action du Sommet mondial de l'alimentation, adopté à Rome le 13 novembre 1996 lors du Sommet mondial de l'Alimentation du 13 au 17 novembre 1996.

${ }^{11}$ La nécessité d'adopter des modes de consommation durables pour assurer une sécurité alimentaire durable a été de nouveau rappelée par la FAO lors de la Conférence mondiale des Nations unies sur le développement durable, dite « Rio + $20 »$, les 20-22 juin 2012.
} 
L'évolution des modes de consommation vers une consommation dite «durable » fait partie des priorités en matière de politique environnementale ${ }^{12}$. Bien qu'il y ait un consensus sur la nécessité de promouvoir et de développer la consommation durable, le concept reste flou et ses contours mal définis. Par ailleurs, le consommateur, acteur majeur de la consommation durable, reste un être paradoxal, dont il est parfois difficile d'appréhender les actes écologiques qu'il est prêt à adopter. Une question fondamentale se pose alors, les choix opérés par les consommateurs résultent-ils de choix personnels ou de comportements influencés par des pratiques habituelles, par l'entourage? Afin d'apporter des éclaircissements à cette interrogation, le Groupe de recherche sur la consommation durable (GRECOD) a mené une étude sur les comportements de consommation dits «verts » ou les profils potentiellement écologiques des ménages français. Grâce à une enquête de grande ampleur auprès d'un panel de consommateurs représentatifs, cette étude a caractérisé les différents profils de consommation durables en termes de critères d'achat, de préférences et de modes de consommation.

\section{Définition de la consommation durable}

1. La consommation durable, un concept consensuel

L'expression «consommation durable » est composée de deux termes a priori antinomiques. Le terme «consommer» vient du latin consummare, qui signifie achever, alors que le terme «durable» exprime une vocation à durer longtemps. La consommation, terme issu de la science économique, est définie comme un ensemble de comportements réalisés par les individus, les entreprises ou l'État dans le but de satisfaire des besoins, depuis la décision d'achat jusqu'aux interactions amont ou aval qui entourent l'acte d'achat et son usage. Même si les économistes classiques, tels que Adam Smith ou Jean-Baptiste Say, soulignent le rôle de la consommation comme but de toute production, il faut attendre le début du $\mathrm{XX}^{\mathrm{e}}$ siècle et, notamment, Thorstein Veblen pour avoir une vision globale de la consommation dans le

\footnotetext{
${ }^{12}$ Voir le Plan d'action pour une consommation, une production et une industrie durables adoptée par la Commission européenne le 16 juillet 2008.
} 
système capitaliste en tant qu'instrument d'imitation sociale, mais aussi en tant que source potentielle de gaspillage ${ }^{13}$.

La consommation durable met l'accent sur la nécessité d'adopter des modes de consommation durables pour assurer à la fois un développement économique pérenne et la protection de l'environnement dans l'intérêt des générations présentes et futures. Le concept de consommation durable demeure encore flou. Toutefois, un consensus émerge quant à sa définition. La consommation durable peut être définie comme «l'utilisation de services et de produits qui répondent à des besoins essentiels et contribuent à améliorer la qualité de la vie tout en réduisant au minimum les quantités de ressources naturelles et de matières toxiques utilisées, ainsi que les quantités de déchets et de polluants tout au long du cycle de vie du service ou du produit, de sorte que les besoins des générations futures puissent être satisfaits $\gg^{14}$.

Cette définition met bien en évidence les différentes dimensions de la consommation durable, qui s'inscrit dans une perspective de satisfaction des besoins individuels, mais également collectifs. Cependant, il demeure difficile de définir plus précisément en quoi consistent les modes de consommation durables et quelles sont les orientations attendues au niveau de l'évolution de la demande des consommateurs. Faut-il consommer moins et/ou différemment?

La question de la consommation durable est souvent envisagée du seul point de vue environnemental, notamment celui des ressources. Pourtant elle ne se limite pas à une consommation écologiquement viable, et inclut aussi les dimensions économiques et sociales du développement durable. Par exemple, l'Agenda 21 adopté lors du Sommet de la Terre en 1992, reconnaît expressément qu' «il existe un lien étroit entre la pauvreté et la dégradation de l'environnement $»^{15}$. Or, la politique européenne de consommation durable promue dans le Plan d'action ne mentionne pas le volet social de la consommation durable.

La dégradation de l'environnement par les modes de consommation des consommateurs, au sens de consommateur final, ne doit pas être négligée. La consommation des consommateurs, considérés en tant qu'acteurs finals, comprend la sélection, l'achat, l'utilisation, l'entretien, la

\footnotetext{
${ }^{13}$ T. VEBLEN, The Theory of the Leisure Class, New York, Macmillan, 1994, 256 p.

${ }^{14}$ Symposium on Sustainable Consumption, Oslo, Norvège, 19-20 janvier 1994.

${ }^{15}$ Agenda 21, chapitre 4 «Modification des modes de consommation ».
} 
réparation et la disposition juridique (abusus) d'un produit ou d'un service. C'est dire que la consommation durable envisage les produits du berceau à la tombe (from craddle to grave) ${ }^{16}$. Les modèles de consommation durable adoptés par les consommateurs sont influencés par différents facteurs qu'il est important d'identifier afin de mieux appréhender le concept de consommation durable.

2. Les facteurs influençant la consommation durable

Au-delà des variables classiques comme l'âge, le revenu, le genre ou la localisation, on peut noter plusieurs dynamiques sociales qui influencent la consommation durable. Ainsi, ce sont les pratiques habituelles qui déterminent le choix des ménages dans leur acte quotidien sans que ces derniers en aient forcément conscience. L'imitation joue aussi un rôle déterminant dans nos pratiques habituelles et reste un moteur de nos actes de consommation quotidienne.

\section{a. Les habitudes et la bonne volonté}

De nombreuses recherches antérieures sur la consommation insistent sur la motivation des individus et les difficultés à mettre en pratique ces bonnes intentions. Il ne suffit pas de vouloir changer les habitudes en matière de consommation énergétique ou alimentaire ou autre. Encore faut-il pouvoir effectivement mettre ses bonnes intentions en pratique, en avoir «l'opportunité » et la capacité de le faire. En effet, les habitudes ont un caractère autorenforçant et sont le fruit d'un apprentissage passé (dimension synchronique des habitudes) et ont aussi un caractère diachronique. Dans le domaine de la consommation durable, plusieurs habitudes peuvent être activées de manière simultanée. Ainsi, il existe à la fois une force des pratiques habituelles liées à leur installation dans le temps mais aussi une interdépendance des actions entre elles. Ainsi à titre d'exemple, la localisation va influencer le mode de transport qui va influencer le type de trajet domicile travail qui va lui-même influencer la nature de la consommation et le choix de l'approvisionnement en supermarché ou ailleurs, etc.

\footnotetext{
${ }^{16}$ Ainsi, selon l'ADEME et Recyc-Québec, alors que le niveau de développement est quasiment équivalent en France et au Canada, un français produit en moyenne $1 \mathrm{~kg}$ de déchets par jour, contre 1 , $69 \mathrm{~kg}$ pour un québécois. Voir ADEME, Les déchets en chiffres en France, ADEME, Paris, 2009 ; Recyc-Québec, Bilan 2006 de la gestion des matières résiduelles au Québec.
} 
Il existe ainsi souvent au sein des individus des bonnes intentions pour mettre en place des changements, toutefois dans la pratique ces formes de motivations se heurtent à la double nature des habitudes. Changer les pratiques habituelles nécessite donc d'aller au-delà de la seule diffusion de d'information et requiert d'observer les ressorts déclencheurs de ces dernières.

b. Le poids de l'imitation dans la consommation

Des résultats empiriques obtenus en psychologie et en économie révèlent une autre facette de cette même dynamique : la volonté des individus d'affirmer leur position relative. Peu importe la nature même de cette consommation dans son contenu, son positionnement relativement aux autres est crucial. Ainsi, il suffit qu'une certaine partie des classes «super aisées » s'enrichisse pour que soit drainée avec elle de nouvelles normes de «standing» véhiculées par les «presque riches ». La dynamique sociale au niveau de l'imitation est majeure. En effet, nous consommons et sommes insérés dans un groupe social. Les valeurs véhiculées par ce groupe et son voisinage auront donc un impact critique sur notre consommation actuelle par effet de contagion. Néanmoins, les influences et les imitations ne sont pas seulement guidées par des valeurs sociales, il peut exister une simple conformité sociale, c'est-à-dire tout simplement la volonté de faire comme les autres et comme notre entourage. Les différents mécanismes d'imitation ne vont pas tous dans la même direction (l'objectif n'est pas toujours de vouloir ressembler aux classes aisées, il peut exister aussi une dynamique inverse).

L'apprentissage de nouveaux comportements, loin d'être trivial, peut néanmoins renverser les «verrouillages comportementaux» initiaux autour de groupes de consommation, dits «verts ». Dans ce contexte, de nouvelles représentations culturelles peuvent faire basculer les modes de consommation traditionnels. La consommation «verte» qui se distinguait auparavant peut donc, sous certaines conditions, se banaliser et devenir un modèle dominant si ce groupe arrive à créer des «biais de conformité » poussant les autres à adopter ces nouvelles formes de consommation. Changer de consommation repose donc tant sur des paramètres économiques entre différents groupes de consommateurs, que sur la capacité de certains groupes à véhiculer des nouvelles valeurs et à fédérer de nouveaux paramètres. 
Le consommateur reste un individu difficile à appréhender. Ses comportements ne se présentent pas toujours en adéquation avec ses déclarations. Au-delà de la terminologie, le consommateur avec ses propres limites sociales et sa propre motivation individuelle doit réaliser divers compromis entre ses intentions, sa capacité d'action, ses désirs et l'environnement institutionnel dans lequel il se situe.

\section{Le consommateur, un acteur majeur de la consommation durable}

Le terme consommateur appartient à la fois au langage commun, au lexique juridique ${ }^{17}$ et à l'économie. Le consommateur est «celui qui détruit un bien ou en détruit la substance, l'utilité » ou encore « le quatrième pôle d'un cycle économique global, au déroulement duquel la consommation, fonction économique, plutôt qu'acte technique, se trouve directement liée $»^{18}$. Toutefois, le concept de consommation durable permet de relier les intérêts économiques des consommateurs, et les valeurs altruistes recherchées par les « consommateurs-responsables » ou les «consommateurs-citoyens », termes fréquemment employés de manière interchangeable. La terminologie «consommateur-citoyen » est fréquemment utilisée pour désigner le consommateur qui rend cohérent ses aspirations citoyennes et ses désirs matériels ${ }^{19}$. La consommation « est devenue un espace de contestation sociale $»^{20}$. En effet, chaque consommateur peut user de son pouvoir économique pour soutenir ou bien refuser certains biens et services selon s'ils correspondent à ses préoccupations environnementales et sociales. L'acte de consommation est alors considéré comme un moment de prise de conscience sociétale. Le comportement des consommateurs est difficilement prévisible. Le consommateur apparaît alors comme un être paradoxal, dont les actes d'achats s'inscrivent à la fois dans une logique hédoniste mais aussi de défense de l'intérêt général ${ }^{21}$.

\footnotetext{
${ }^{17}$ G. CORNU, Linguistique juridique, Paris, Montchrestien, 1990, p. 68 s.

${ }^{18}$ T. BOURGOIGNIE, Eléments pour une théorie du droit de la consommation : au regard des développements du droit belge et du droit de la Communauté économique européenne, Bruxelles, Bruylant, 1988, $34 \mathrm{~s}$.

19 T. WILHELMSSON, «Consumer Law and the Environment: From Consumer to Citizen », Journal of Consumer Policy, 1998, n² 21, p. 45.

${ }^{20}$ S. DUBUISSON-QUELLIER, La consommation engagée, Paris, Les Presses De Sciences Po, 2009, p. 11.

${ }^{21}$ S. LANGLOIS, Consommer en France : cinquante ans de travaux scientifiques au Crédoc, Paris, Editions de l'Aube, 2005, p. $6 \mathrm{~s}$.
} 
Il convient dès lors de se demander si les consommateurs ont la possibilité d'agir de façon responsable et durable sur les marchés à travers notamment leurs actes d'achat. Il est donc essentiel que les contractants, ici aux consommateurs, s'engagent librement en pleine connaissance de cause. On constate qu'un nouveau droit se met en place pour réconcilier l'homo economicus et le citoyen. Celui-ci entend «voter avec son porte-monnaie », c'est-àdire se saisir du marché comme lieu d'action politique ${ }^{22}$. Ce mouvement semble correspondre à un moment où les usages politiques se renouvellent partout dans un mouvement de réappropriation individuelle du politique et où, dans la sphère de la consommation, les préoccupations environnementales, sociales, de santé ${ }^{23}$ et éthiques montent en puissance. Le succès de l'agriculture biologique et du commerce équitable constitue à cet égard une preuve tangible de ce que les consommateurs sont de plus en plus attentifs au lien entre les produits et services qu'ils achètent et les enjeux fondamentaux attachés aux lieux et aux conditions (sociales et processus de production) de leur production ${ }^{24}$. Cette nouvelle forme d'engagement politique des consom'acteurs ${ }^{25}$ soulève bien évidemment la question de l'équité dans les formes de consommation ${ }^{26}$. On peut, en effet, se demander si les formes de consommation durable ne sont destinées qu'à rester l'apanage des plus riches. Le risque de cette nouvelle forme d'engagement citoyen via le marché est peut-être celui de la dissolution du politique dans le marché et d'une récupération par le marketing ${ }^{27}$.

\footnotetext{
${ }^{22}$ S. DUBUISSON-QUELLIER, La consommation engagée, Paris, Les Presses De Sciences Po, 2009, p. 11 ; M. MICHELETTI, «Consommateurs et citoyenneté ", in Repenser l'action de consommation pour le bien-être de tous. Réflexions sur la responsabilité individuelle des consommateurs, Strasbourg, Conseil de l'Europe, 2008, p. 28.

${ }^{23}$ F. COLLART DUTILLEUL, «Le consommateur face au risque alimentaire. Pour une mise en œuvre raisonnable du principe de précaution », in J. CALAIS-AULOY (dir.), Etudes de droit de la consommation, Liber amicorum J. Calais-Auloy, Paris, Dalloz, 2004, p. 311.

${ }^{24}$ Nous ne pouvons dans ce travail développer l'opposition frontale qui existe et qui s'est manifestée à l'OMC entre les Etats-Unis et l'Union européenne sur la question des processus de production.

${ }^{25}$ A propos de la notion de «consom'acteur », voir S. MAHIEU, Le droit de la société de l'alimentation : vers un nouveau modèle de maîtrise des risques alimentaires et technologiques en droit communautaire et international, s'intéresse particulièrement à la notion de " consommacteurs », Larcier, Bruxelles, 2007, 674 p.

${ }^{26}$ M. P. SORENSEN, «Economie, consumérisme et démocratie libérale », Courrier de la planète, 2009; M. FIGUIE et N. BRICAS, «Equité internationale : la surresponsabilisation des consommateurs », Courrier de la planète, 2009, n ${ }^{\circ} 87$, p. 41 : «En laissant le marché se faire l'expression des projets de société, il n'est pas sûr que les citoyens s'y retrouvent. Et cette forme d'engagements pourrait bien provoquer des effets inverses à ceux que souhaitent les citoyens ».

${ }^{27}$ L'association écologique, Les Amis de la Terre, attribue tous les ans le prix Pinocchio du développement durable qui vise à dénoncer le greenwashing mené par certaines entreprises françaises.
} 
À travers les outils de régulation possible mis en place par le législateur, on voit bien deux types de consommation durable, une version faible et une version forte ${ }^{28}$. Dans le premier cas, les consommateurs font évoluer graduellement leurs modes de consommation pour intégrer des valeurs et pratiques environnementales. Il s'agit soit d'acheter les fruits et légumes de saison, soit de se conformer aux labels existants pour changer les réflexes de la consommation courante en y intégrant de nouveaux critères de choix. Cette nouvelle forme de consommation est à l'heure actuelle la plus fréquemment adoptée et repérée dans les enquêtes de consommation en France et dans l'ensemble des pays industrialisés (environ 20 à $30 \%$ de consommateurs sont prêts à intégrer ces variables dans leur choix ou les réalisent déjà en France). La plupart du temps, ces consommateurs disposent d'une bonne information, mais aussi de revenus suffisants pour rendre cohérents leurs choix avec leurs propres contraintes budgétaires.

La version faible de la consommation durable permet ainsi de consommer mieux en incluant un certain nombre de pratiques (tri des déchets, par exemple), de motivations individuelles et de valeurs dans la consommation finale des ménages. Ceci n'exclut pas un ensemble de contradictions inhérentes aux choix et modes de vie des ménages. En effet, dans cette version faible, les modes de consommation ne sont pas fondamentalement remis en cause si bien que l'on peut avoir des consommateurs ayant des pratiques de consommation durable tout en utilisant, par exemple, des modes de transports privés. Si ces incohérences subsistent, elles demeurent néanmoins un premier pas avant de réaliser une refonte de modes de consommation usuels.

La consommation durable, version forte, implique un changement plus radical visant à réduire la consommation finale. Les consommateurs consomment différemment et réduisent certaines formes d'achats. La mise en place de nouvelles formes de services est ainsi préconisée dans cette version, notamment pour accélérer l'usage de transports collectifs, mais également pour encourager le recours à de nouvelles formes de services (type location au lieu d'achat). Cette version forte implique de considérer sur le long terme la pratique d'achat et de l'envisager dans sa globalité pour aller au-delà de l'acte de consommation et résoudre certaines formes de contradiction. L'« effet rebond » illustre cette possible incohérence dans nos comportements.

\footnotetext{
${ }^{28}$ D. A. FUCHS et S. LOREK, « Sustainable consumption governance: a history of promises and failures », Journal of Consumer Policy, 2005, n² 28, p. 261.
} 
Il consiste, en effet, à consommer certaines formes de biens moins «énergétivores », mais à augmenter la consommation d'énergie finale. Il s'agit, par exemple, des voitures moins polluantes qui sont utilisées plus souvent ou bien de l'achat d'un réfrigérateur acheté en fonction de la classe énergétique réduite, mais qui se réalise conjointement avec la conservation de l'ancien, ce qui a pour résultat d'augmenter la consommation finale d'énergie. Dans la version forte, la révision du modèle de consommation globale est réelle.

En fait, l'ensemble des études montre que peu de consommateurs sont prêts à aller dans cette direction plus contraignante. Cette dernière émerge toutefois dans certaines formes de groupements sociaux ou politiques à travers, par exemple, la floraison du mouvement des Associations pour le maintien d'une agriculture paysanne (AMAP) remettant en cause nos modèles de consommation et de production par la mise en place de nouveaux liens entre agriculteurs et ménages. Dans la version forte, l'achat de services est donc privilégié ainsi que de nouvelles formes de liens sociaux. Dans les «éco quartiers», de nouvelles formes de gouvernance peuvent ainsi mettre en place une réflexion sur la consommation et la ville durable (comme on l'obverse pour la ville de Nantes).

Il ressort donc que la consommation durable, dans sa version forte, doit pour se pérenniser s'inscrire sur de nouvelles formes collectives d'actions qui ne sont plus uniquement juridiques. En effet, cela nécessite aussi, au-delà de l'acte de consommation, de repenser les infrastructures et les modes d'aménagement du territoire. Le législateur n'est plus le seul acteur, mais sert de relais à de nouvelles formes d'organisations sociales qui concourent à imaginer la consommation et la ville de demain. Cette réflexion de grande ampleur nécessite, au-delà du droit de la consommation, des expérimentations locales pour amorcer une révision de notre modèle de consommation comme la volonté des politiques locales, nationales et internationales. Bref, une réflexion de grande envergure qui aboutit à de nombreuses préconisations et textes internationaux dont le degré d'application fluctue selon les sensibilités et les traditions des ménages et leur environnement politique et social.

Les pouvoirs publics et les entreprises privées doivent favoriser l'adoption de modes de consommation durables par les consommateurs. Cependant, les consommateurs ne doivent pas sous-estimer leur propre capacité à agir individuellement. L'enquête menée par le GRECOD sur le territoire français permet de caractériser les différents profils des 
consommateurs écologiques en termes de critères d'achat, de préférence et de modes de consommation.

\section{Caractérisation des profils de consommateurs écologiques}

\section{Contexte de l'étude empirique}

Dans le cadre du programme «Déchets et Sociétés » financé par l'ADEME, un groupe de chercheurs français, le GRECOD (Groupe de Recherche sur la Consommation Durable), a procédé à une étude sur les comportements de consommation dits «verts » ou écologiques ${ }^{29}$. Auprès d'un panel de consommateurs représentatifs de la France entière (représentativité au sens de l'INSEE), le GRECOD a caractérisé différents profils en termes de critères d'achat, de préférences et valeurs. L’échantillon porte sur plus de 3024 ménages en France.

L'objectif de l'enquête menée auprès des ménages français était de caractériser différentes pratiques afin d'appréhender la consommation dans sa globalité, notamment la question du tri des déchets. Plusieurs pratiques ont été examinées: le tri, les pratiques énergétiques (consommation d'énergie, achat d'énergie renouvelable, consommation énergétique lors de l'utilisation du lave-linge), les pratiques alimentaires (critères de choix de manière générale et petit focus sur le yaourt) et le mode de transport.

Plusieurs variables comportementales ont été prises en compte pour caractériser les pratiques des consommateurs, par exemple, l'imitation sociale et l'influence du voisinage lors de certaines pratiques ; les valeurs des ménages (capacité à identifier des priorités sur des dons éventuels à une association environnementale, de santé ou autre); et les critères déterminants pour définir la consommation durable et la protection de l'environnement.

Les précédentes enquêtes menées en France et à l'étranger montrent qu'au niveau des obstacles vers le «verdissement» des comportements, les ménages à titre individuel déclarent que la variable prix reste le principal frein. Au niveau des achats alimentaires, l'achat de

\footnotetext{
${ }^{29}$ Groupe de REcherche sur la COnsommation Durable : Une étude des profils de consommation écologiques, rapport final Décembre 2012, Programme «Déchets et Société » pour le compte de l'ADEME. Les auteurs sont Jean Belin, Ali Douai, Sébastien Lavaud, Nathalie Lazaric, Fabrice le Guel et Vanessa Oltra..
} 
produit bio ou « respectueux de l'environnement » doit d'abord entrer dans le quotidien avant l'achat. Une certaine familiarité (à travers l'achat de proximité) permet de prendre l'habitude d'acheter ces produits «verts» et de les repérer dans les centres différents centres de distribution. Ainsi, le manque de réflexe ${ }^{30}$ et le fait que ces produits ne soient pas directement accessibles dans leur contexte habituel constituent des freins aux comportements écologiques des consommateurs ${ }^{31}$. Enfin, des recherches réalisées en Allemagne insistent sur un des éléments préalable pour initier la consommation durable ${ }^{32}$, à savoir l'imitation des autres dans certains groupes sociaux. les chercheurs allemands montrent ainsi comment certains « groupes de référence » diffusent de nouvelles pratiques habituelles aux autres membres du groupe, appelés les «influents». Par ailleurs, l'entourage social a une influence sur les individus et peut faire infléchir leur motivation intrinsèque (motivation autonome et individuelle), voire les valeurs de certains consommateurs sur une longue période ${ }^{33}$. Le fait d'être inséré dans un groupe ou dans un réseau aurait donc une réelle influence et un effet démonstration sur les pratiques individuelles.

\section{Résultats de l'enquête}

L'enchâssement social est notable dans la caractérisation des profils de consommation écologique, c'est-à-dire que la consommation durable est fortement dépendante des pratiques habituelles de l'entourage. Il est donc essentiel que les politiques environnementales prennent d'avantage en compte la dimension collective de la consommation durable.

Cet effet de l'entourage est évalué notamment à travers la question portant sur la présence dans le voisinage de personnes qui sont sensibles à l'environnement (Avez-vous dans votre entourage (famille / amis) des personnes qui prennent en compte les effets sur l'environnement des produits qu'elles achètent?). La dimension collective de la consommation durable existe dans différentes pratiques: le tri des déchets, les pratiques alimentaires, l'utilisation du lave-linge, les pratiques énergétiques et les transports.

\footnotetext{
${ }^{30}$ Enquête menée par Agence bio en 2011.

${ }^{31}$ Enquête menée par le cabinet Atefo, Mouvement Vraiment Durable, en 2007.

32 H. WELSCH et H., KÜHLING, J., 2009, "Determinants of pro-environmental consumption: The role of reference groups and routine behavior, Ecological Economics, Elsevier, vol. 69(1), pages 166-176, November. ${ }^{33} I d$.
} 
a. Importance de la dynamique sociale dans les pratiques environnementales

Bénéficier d'un entourage conscient de ses impacts environnementaux, être personnellement sensible à l'environnement sont autant d'éléments qui peuvent être considérés comme des caractéristiques favorisant les pratiques durables. Néanmoins, il n'existe pas de lien entre les sensibilités environnementales et le fait de trier. Pour les pratiques habituelles de tri, il faut chercher dans d'autres variables comme la localisation géographique.

b. Effet âge, genre et statut matrimonial

Conformément aux résultats des études précédentes, l'analyse des résultats de l'enquête GRECOD permettent de dégager des effets genre, âge, éducation et statut matrimonial. Ainsi, appartenir à une classe d'âge plutôt jeune (de 18 à 35 ans) peut être considéré comme un frein, un obstacle à l'adoption de pratiques durables pour le tri et l'alimentation. A l'inverse, les classes plus âgées se caractérisent par l'adoption de pratiques plus économes dans le domaine de l'énergie. Une analyse complémentaire montre que la probabilité d'opter pour des pratiques alimentaires durables est faible pour les individus les plus jeunes et significative pour les individus autour de 55 ans puis tendrait à décroître significativement après 70 ans.

c. Importance de l'âge et du statut dans les pratiques environnementales

L'effet genre et le statut (marital ou non) sont importants pour l'adoption de pratiques environnementales. Ainsi, être un homme et être célibataire constituent des freins à l'adoption de pratiques alimentaires, énergétiques et globales durables.

Un effet éducation se manifeste pour l'ensemble des pratiques à des degrés plus faibles : ne pas être ou n'être que faiblement diplômé tend à être une «dés incitation » à l'adoption de comportements de consommation durable. 
d. Des effets plus ambigus : effet région, effet agglomération

Les résultats de l'enquête GRECOD mettent en avant une influence de la région sur certaines pratiques. On constate que l'appartenance au groupe ayant les pratiques globales les plus durables est effectivement favorisée par les caractères régionaux et que les 8 grandes subdivisions du territoire métropolitain ont une portée explicative plus ou moins marquée. Résider dans l'Est de la France influence positivement l'adoption de pratiques durables. Les régions Ouest et Sud-Ouest présentent un indéniable effet facilitateur de comportement de consommation durable pour l'alimentaire et les pratiques globales.

Enfin, la taille de l'agglomération peut également constituer un frein à l'adoption de pratiques durables. On constate ainsi que cet effet est très significatif pour les unités urbaines de plus de 200000 habitants.

Les résultats de l'enquête GRECOD soulignent l'importance des fortes disparités régionales entre les différentes pratiques durables. La région méditerranée est, à cet égard, riche d'enseignement car elle recèle d'un ensemble de bonnes pratiques au niveau alimentaire que l'on ne retrouve pas au niveau du recyclage et du tri. Il semblerait donc pertinent de mieux comprendre les ressorts de ces comportements, notamment en matière de non tri, par des approches qualitatives ou quantitatives complémentaires (poids des infrastructures, émulation sociale jouant négativement et autres variables). La région parisienne semble aussi peu orientée vers des pratiques dites durables (effet conjugué du caractère urbain et de la présence de nombreux célibataires).

Les résultats obtenus en termes statistiques démontrent indéniablement l'importance des pratiques d'imitation sociale qui priment sur toute autre dynamique. Il pourrait donc être pertinent de repérer des régions motrices en matière de pratiques durables dans l'Ouest et le Sud-Ouest de la France pour en tirer des modèles de cercle vertueux dont la France pourrait bénéficier. Par ailleurs, il serait intéressant d'analyser des externalités positives agissant comme moteur et modèle de références pour d'autres consommateurs. Cette dynamique est située en dehors des villes et dépend de l'histoire locale et régionale inhibant ou au contraire propulsant certaines régularités comportementales dont les individus n'ont pas toujours 
conscience. Mieux localiser ces pratiques durables et mieux identifier leur histoire et leurs ressorts permettrait d'appréhender d'avantage les pratiques de consommation durable adoptées par les consommateurs écologiques.

Dans les pays occidentaux, le consommateur d'aujourd'hui est un être paradoxal dont il reste difficile d'appréhender le comportement écologique. Cependant, les consommateurs ne doivent dès lors pas sous-estimer leur propre capacité à agir individuellement. Par exemple, dans les caddies de supermarchés, les produits ordinaires côtoient les produits issus du commerce équitable et ceux issus de l'agriculture biologique. Les consommateurs se servent de sacs de caisse réutilisables, tri sélectif, achat de produits d'occasion sur des sites internet bien connus ; etc. Les outils de régulation au niveau de la consommation existent notamment les outils fiscaux mais ils ont leurs propres limites étant donné les compromis que réalisent les consommateurs (étant donné leur genre, leur localisation et leur statut marital ou non). Une chose est sûre, la consommation durable ne se décrète pas mais s'apprend. Le régulateur et le décideur public doivent donc tenir de cette dimension avant de mettre en place tout nouveau dispositif réglementaire au niveau de la consommation. 